\title{
The Effect of Tracheal Smooth Muscle Tone on Neonatal Airway Collapsibility
}

\author{
VINOD K. BHUTANI, RANDY J. KOSLO, AND THOMAS H. SHAFFER \\ Department of Physiology. Temple University School of Medicine, and Section on Newborn Pediatrics, \\ Pennsylvania Hospital, University of Pennsylvania, Philadelphia, Pennsylvania
}

\begin{abstract}
The effect of smooth muscle tone on the functional characteristics of neonatal airways was studied in six newborn lambs. Tracheal mechanics, resistance, and collapsibility were determined in tracheal segments of uniform length which were surgically isolated just caudal to the cricoid cartilage. Resistance to airflow through uncompressed (zero transmural pressure) and compressed (increased transmural pressure) trachea was evaluated over a range of physiological flows both before and after induced contraction of airway smooth muscle. Results demonstrate that neonatal tracheal smooth muscle does respond to cholinergic stimulation and that there was a significant decrease in airway compliance. Furthermore, the consequent increase in airway rigidity may play a role in resisting collapse or compression of the intrathoracic airway during expiration. (Pediatr Res 20: 492-495, 1986)
\end{abstract}

Structurally, neonatal airways are more compliant than adult airways (1-3). The effect of any transmural compression on these compliant structures could, therefore, lead to increased airflow resistance on expiration and consequently require additional work in breathing. Tracheal smooth muscle plays an important role in regulating the conducting airway volume and resistance in the adult (4). Furthermore, when the tracheal smooth muscle tone is increased $(2,5)$ adult airways (intrathoracic) have been shown to resist compression during expiration. However, although it is known that the trachealis muscle mass increases throughout postconceptional development, little quantitative data are available to confirm the relationship between smooth muscle contraction and mechanical function of the neonatal airways.

This study was designed to evaluate the effect of neonatal airway smooth muscle tone on tracheal mechanics, resistance, and collapsibility. Resistance to airflow through uncompressed (zero transmural pressure) and compressed (increased transmural pressure) tracheae were examined over a range of physiological flows both before and after induced contraction of airway smooth muscle. Changes in airway resistance were associated with alterations in tracheal mechanics due to smooth muscle contraction. These studies should be useful in the understanding of how airway smooth muscle may increase the ability of immature airways to withstand compressive narrowing.

\section{METHODS}

Animal preparation. Six term neonatal lambs (1-5 postnatal days) were sedated with intraperitoneal administration of pen-

Received September 25, 1985; accepted January 23, 1986

Send correspondence and requests for reprints to Vinod K. Bhutani, M.D., Section on Newborn Pediatrics, Pennsylvania Hospital, 8th and Spruce Streets, Philadelphia, PA 19107

Supported in part by NIH Grant HL32031. tobarbital $(20 \mathrm{mg} / \mathrm{kg})$. Each was secured in a supine position and the cervical trachea was exposed through a midline incision. The trachea was cannulated close to the thoracic inlet to establish a patent airway for spontaneous ventilation. Care was taken to preserve vascular and neural supply by utilizing blunt dissection. The left jugular vein was catheterized to administer drugs and dextrose infusion. The electrocardiogram was continuously monitored for lead II. The lambs were maintained normothermic under a heat lamp. The bypassed tracheal segment was utilized to determine pressure-volume and pressure-flow relationships. Throughout the experiment, the segment was covered with moist dressing to prevent dessication. The tracheal length was fixed in a rigid frame between the proximal and distal ligatures at about $120 \%$ of its resting length (6).

Pressure-volume relationships. In vivo tracheal mechanics were determined in the bypassed cervical tracheal segment. The proximal end was cannulated and connected to a calibrated Hamilton microsyringe and an injector system. The distal tracheal end was connected to a micropipette and a pressure transducer (Statham p23 db). The entire system was filled with isotonic saline and made leak free.

The baseline intratracheal pressure of the fluid-filled segment was recorded at ambient and zero transmural pressures. The segment was then closed to the atmosphere. The volume injected through the microsyringe to achieve an intratracheal pressure of $80 \mathrm{~cm} \mathrm{H}_{2} \mathrm{O}$ was recorded for each lamb. To obtain pressurevolume relationships, $25 \%$ of this volume was injected at $15-\mathrm{s}$ intervals. Concurrent intratracheal pressures were recorded for each volume increment. Similarly, volume was withdrawn in $25 \%$ decrements and quasistatic pressure-volume relationships were obtained for each tracheal segment.

Pressure-flow relationships. The tracheal segment was drained of the isotonic saline. Flow through the segment was achieved by attaching a vacuum source to the distal end. Approximate assessment of flow was measured by a pneumotach (Fleish no. 01 ) and accurately determined by a Collins' spirometer. The pressure change across the bypassed segment was measured by a Statham PM 131TC differential pressure transducer; the intratracheal pressure was determined by the Statham p $23 \mathrm{db}$ transducer. Airflow through the tracheal segment was varied from 5 to 30 liter $/ \mathrm{min}$. A variable resistor, in series with the flow, was utilized to control the transmural pressure from 0 to $-40 \mathrm{~cm}$ $\mathrm{H}_{2} \mathrm{O}$. The differential pressure across the tracheal segment for tracheal airflows of 5,15 , and $30 \mathrm{liter} / \mathrm{min}$ was measured individually at transmural pressures of $0 \mathrm{~cm} \mathrm{H}_{2} \mathrm{O}$ (uncompressed) and at $-40 \mathrm{~cm} \mathrm{H}_{2} \mathrm{O}$ (compressed). Differential pressure-flow relationships at these two transmural pressures were constructed for each tracheal segment.

Tracheal smooth muscle stimulation. A cholinergic agonist, carbamyl-methylcholine (Bethanecol) was utilized to elicit tracheal smooth muscle contraction. Preliminary studies in our laboratory demonstrated that a dosage of $0.16 \mathrm{mg} / \mathrm{kg}$ in the newborn lamb resulted in maximal airway muscle stimulation 
which persisted for $3 \mathrm{~h}$ with minimal cardiovascular effects (a transient decrease in heart rate $10-15 \mathrm{~s}$ duration).

On completion of the control recordings of pressure-volume and pressure-flow relationships, the tracheal segment was again filled with isotonic saline and attached to a micropipette. Bethanecol was injected through the jugular vein. The change in tracheal volume consequent to tracheal constriction was measured by the micropipette. Tracheal mechanics were now redetermined in the stimulated state as described earlier in the text.

Tracheal dimensions. The fixed length of the tracheal segment was determined as the distance between proximal and distal ends of the frame. The circumference of the segment was measured in situ during the stimulated state on completion of the experiment. Assuming that the segment was cylindrical, these data were utilized to calculate mathematically the tracheal volume. To calculate the volume of the segment in the control state, the volume of saline measured by the micropipette following Bethanecol administration was added to the earlier calculated volume.

Data analysis. Quasistatic pressure-volume relationships were plotted for individual segments in the control and stimulated states. Tracheal compliance was measured as the change in volume for a change in intratracheal pressure. This measurement was determined at 95 to $105 \%$ of control volume and similar isovolumic conditions in the experimental state as shown in Figure 1.

Differential pressure and tracheal air flow relationships were developed at both 0 and $-40 \mathrm{~cm} \mathrm{H}_{2} \mathrm{O}$ transmural pressures. Tracheal resistances and the ratio of differential pressure and tracheal air flow were calculated for each segment before and during cholinergic stimulation, both at rest and at the compressed state. Control and experimental data were compared for significant changes using the Student's $t$ test for paired data.

\section{RESULTS}

The mean control volume of the tracheal segments was calculated to be $3.42 \pm 0.5 \mathrm{SEM} \mathrm{ml}$. After cholinergic stimulation, the tracheal volume decreased by $11.6 \%(p<0.01)$. Pressurevolume relationships of a tracheal segment before and during cholinergic stimulation are shown in Figure 1. Values for tracheal compliance about isovolumic point are tabulated in Table 1. A significant decrease $(55 \%, p<0.02)$ in tracheal compliance was observed as a result of increased tracheal smooth muscle tone.

Typical pressure-flow relationships during tracheal relaxation and tracheal collapse, before and during cholinergic stimulation, are illustrated in Figure 2. In the controlled state, the tracheal pressure increased linearly as tracheal airflow increased. However, with tracheal compression, the increase in the tracheal segmental pressure was exponential with increased airflow. After cholinergic stimulation, the increase in tracheal pressure was uniformly linear, even during tracheal compression.

The changes in tracheal resistance after tracheal smooth muscle stimulation are illustrated in Figure 3. Tracheal resistances are shown as a result of Bethanecol stimulation and a transmural pressure of 0 and $-40 \mathrm{~cm} \mathrm{H}_{2} \mathrm{O}$, at flow rates of $5,15,30$ liter/ min. These data demonstrate that following tracheal muscle contraction at zero transmural pressure, a minimal decrease in tracheal resistance is observed. Tracheal resistance increased

Table 1. Tracheal compliance before and after tracheal smooth muscle stimulation

\begin{tabular}{ccc}
\hline Lambs & Control & Experimental \\
\hline 1 & 0.029 & 0.020 \\
2 & 0.043 & 0.011 \\
3 & 0.022 & 0.008 \\
4 & 0.012 & 0.005 \\
5 & 0.020 & 0.009 \\
6 & 0.034 & 0.009 \\
& & \\
Mean & 0.027 & $0.012^{*}$ \\
\pm SEM & 0.004 & \pm 0.002 \\
\hline
\end{tabular}

* Level of significance $(p<0.01)$.

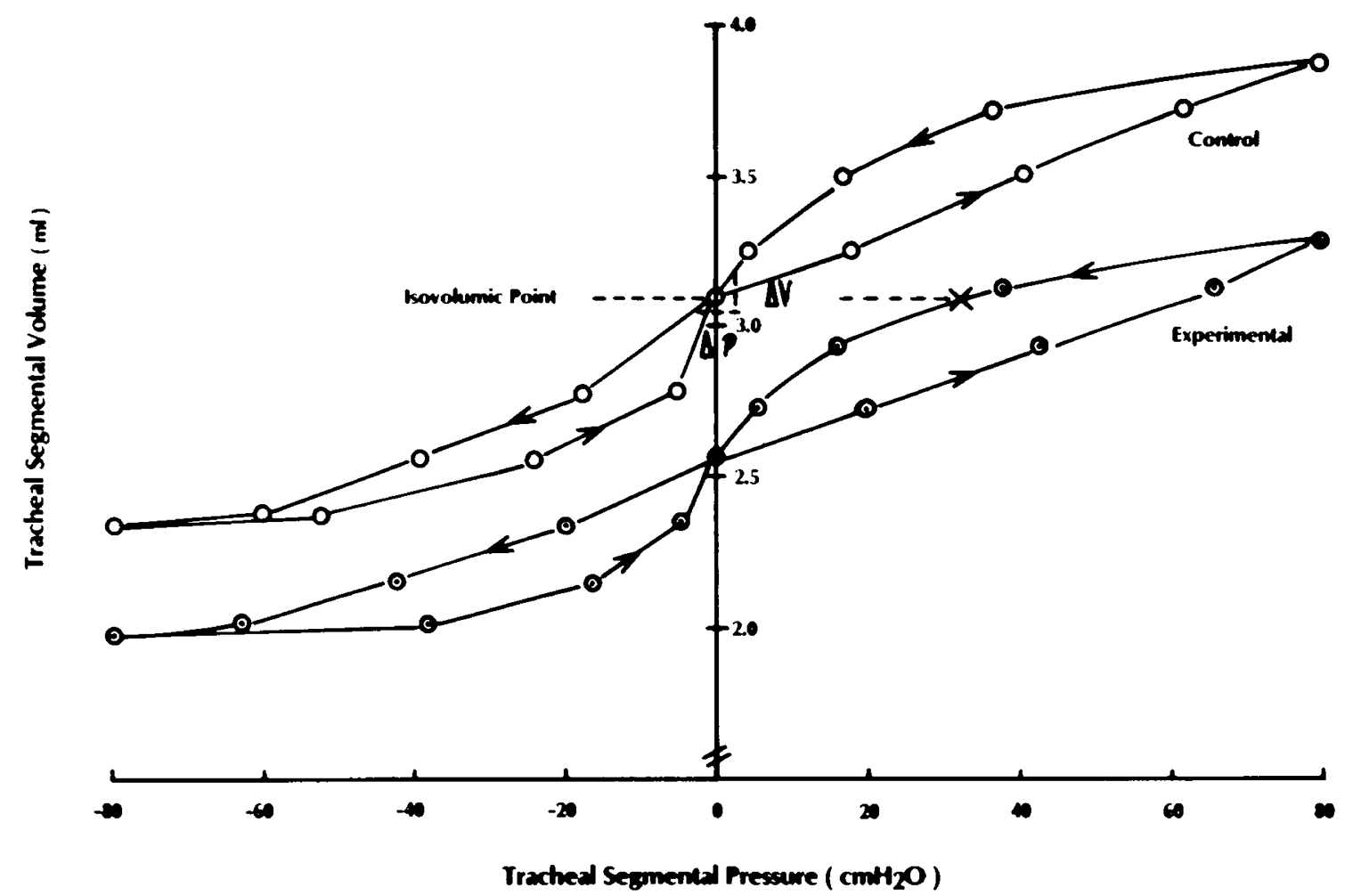

Fig. 1. Pressure-volume relationships of a lamb tracheal segment in both control $(\odot)$ and experimental $(\odot)$ states. Tracheal compliance measured as $\triangle \mathrm{V} / \triangle \mathrm{p}$ at the isovolumic points. Arrows indicate inflation and deflation of the tracheal segment. 

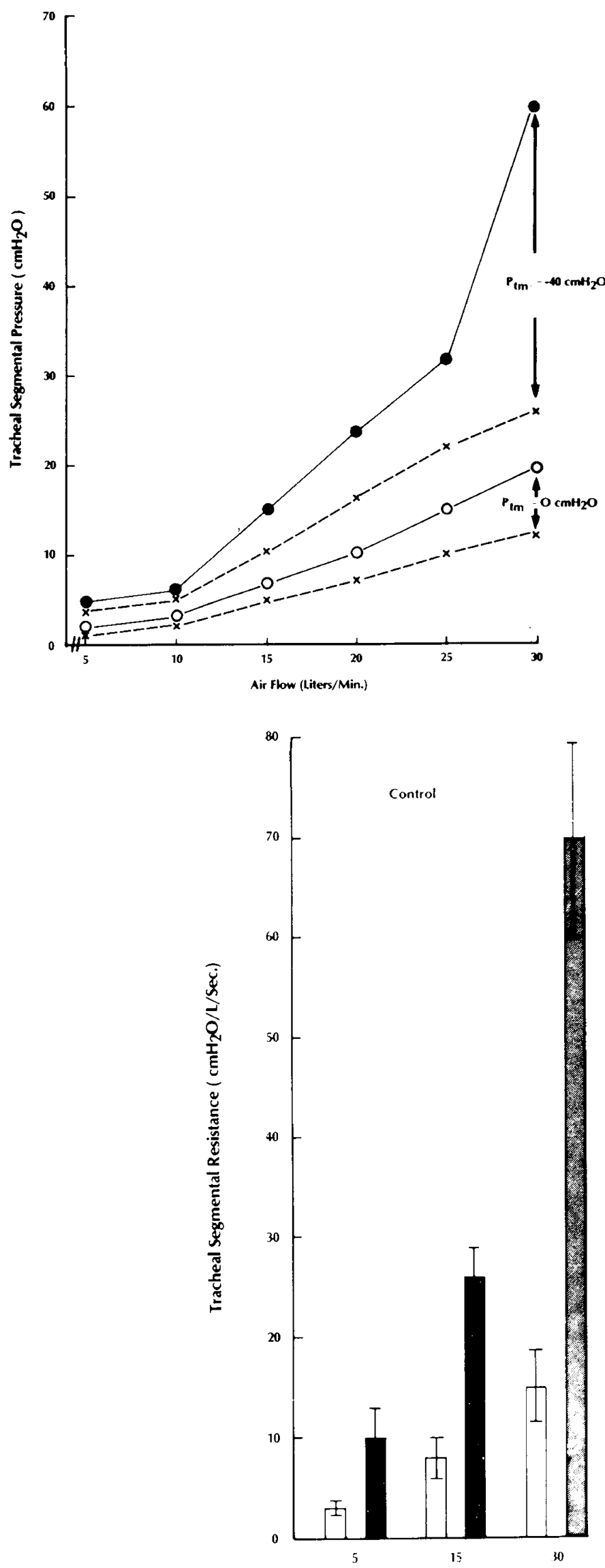

Air Flow (Liters/Min.) significantly with compression (increased collapsing transmural pressures). At $-40 \mathrm{~cm} \mathrm{H}_{2} \mathrm{O}$, a significant decrease $(p<0.02)$ in tracheal resistances was observed at both low and high flow rates following cholinergic stimulation. Mean tracheal resistance was reduced to $61 \%$ of control values when tracheal smooth muscle tone was increased, and the trachea collapsed with the airflow at 30 liter/min. The data in Figure 3 illustrate significant reduction in tracheal resistance at high flow rates (15 to 30 liter/min) and/ or tracheal collapse when cholinergic stimulation is applied.

\section{DISCUSSION}

This study confirms that the collapse of compliant neonatal airways leads to a concomitant increase in their resistance to airflow. An increase in tracheal smooth muscle tone blunts the collapsibility of these airways such that the resistance to airflow is significantly lowered. These observations are particularly evident at airflows of 15 to 30 liter/min.

The compliant and collapsible nature of in vitro preterm airways is well recognized (1-3). Utilizing rabbit tracheal segments we have previously characterized the passive properties of the immature airways (1). Tracheal smooth muscle is tonically

Fig. 2. Pressure-flow relationships of a typical tracheal segment are shown in the control state under no compression $(\mathrm{O})$ and during compression with a transmural pressure of $-40 \mathrm{~cm} \mathrm{H}_{2} \mathrm{O}(\bullet)$. Pressure-flow relationships for the experimental state during both uncompression and compression are shown in dashed lines.

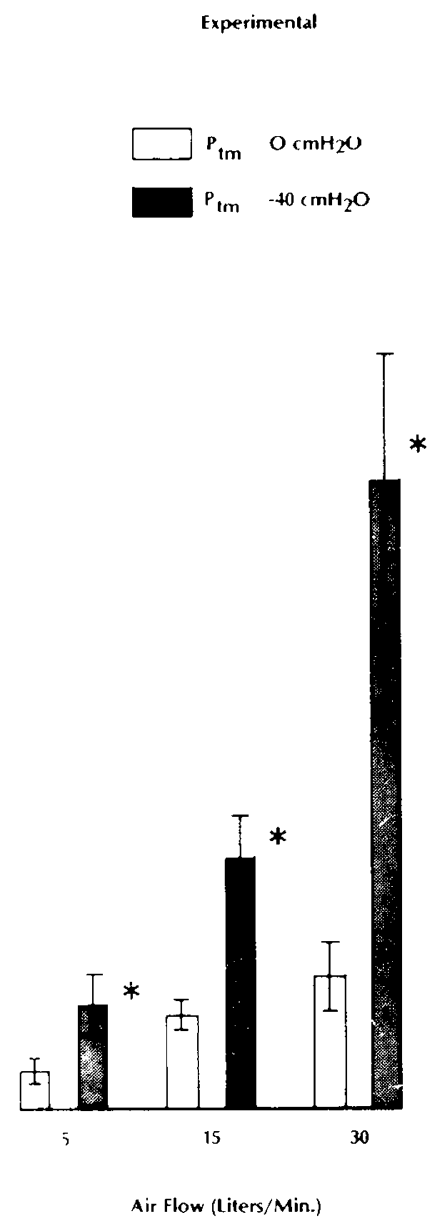

Fig. 3. Tracheal segmental resistances (mean \pm SEM) calculated at airflow rates of 5,15 , and 30 liter/min are shown for both control and experimental states when compressed (solid bars) and uncompressed (open bars). 
active (adult airway studies); however, its effect on the functional behavior of immature airways has not been previously evaluated. In this regard, Palombini and Coburn (7) have documented that graded increases in the active tension of trachealis muscle of adult dogs resulted in a proportional decrease in tracheal compliance. The effect of smooth muscle on adult tracheae have been studied with respect to viscoelastic properties (8), regulation of tone (9), and structure function (10). The tone of the tracheal smooth muscle would be dependent on its mass and on its ability to respond to cholinergic stimulation. Limited data are available on the postconceptional development of tracheal muscle. It is to be noted that tracheal-bronchial smooth muscle hyperplasia does occur consequent to either oxygen-induced or pressure-induced trauma, as is seen in bronchopulmonary dysplasia (11). The present study confirms that in term animals, the tracheal smooth muscle does respond to cholinergic stimulation. Furthermore, the increased smooth muscle tone results in a $55 \%$ reduction of tracheal compliance. These observations are in comparison to a $39 \%$ (7) and $30 \%$ (12) reduction in adult canine tracheal compliance following maximal increase in active tension.

The effect on tracheal mechanics may be attributed to stretching of the series elastic component of the smooth muscle and thus causing a shift toward the stiffer segment of the pressurevolume relationship (13). This shift in operating range is in effect due to a more rigid posterior tracheal wall and fibromuscular rings between cartilaginous plates. As shown for adult airways, this consequent increase in airway rigidity could play a significant role in resisting collapse or compression (12). Furthermore, the surrounding anatomical structures may enhance the stability of the airways. However, the compliance of these structures may itself change as a function of gestational age and maturation. The mechanical properties of the airways do affect maximum expiratory flow $(14,15)$ and resistance to airflow $(2,16)$. Coburn and Palombini (7) have shown that smooth muscle contraction in adult tracheae resulted in increased airflow resistance in the uncompressed state but decreased resistance when compressive pressure was applied (2). Knudson and Knudson (16) have also corroborated these findings. Theoretically, the neonatal airways are more susceptible to collapse during expiration than the more rigid adult airways. Thus, the role of smooth muscle tone in providing greater airway stability and attenuating airway collapse during expiration may be more critical. In the present study, a reduction in tracheal compliance was consistently associated with a reduction in tracheal resistance over a wide range of flow rates and for both the uncompressed and compressed conditions.

In conclusion, this study demonstrates that neonatal airway smooth muscle may provide a mechanism for altering expiratory flow limitation. In addition to its role in regulating dead space volume and airflow resistance, the tracheal smooth muscle tone may play a protective role by resisting collapsibility in relatively compliant airways.

Acknowledgments. The authors thank Ms Phyllis Sparrow for her secretarial assistance and Ms Shalini Dev for her editorial assistance.

\section{REFERENCES}

1. Bhutani VK, Rubenstein SD, Shaffer TH 1981 Pressure-volume relationships of trachea in fetal newborn and adult rabbits. Respir Physiol 43:221-231

2. Burnard ED, Grattan-Smith P, Picton-Warlow, Grauaug A 1965 Pulmonary insufficiency in prematurity. Aust Pediatr $\mathbf{J} 1: 12-38$

3. Croteau JR, Cook CD 1961 Volume-pressure and length-tension measurements in human tracheal and bronchial segments. J Appl Physiol 16:170 172

4. Widdicombe JG 1963 Regulation of tracheobronchial smooth muscle. Physiol Rev 43:1-37

5. Coburn RF, Palombina B 1972 Time-dependent pressure-volume relationships of the in vivo canine trachea. Respir Physiol 16:282-289

6. Coburn RF, Thortin D, Arts R 1972 Effect of trachealis muscle contraction on tracheal resistance to airflow. J Appl Physiol 32:397-403

7. Palombina B, Coburn RF 1972 Control of the compressibility of the canine trachea. Respir Physiol 15:365-383

8. Bobbaers H, Clement J, van de Westigne KP 1978 Dynamic viscoelastic properties of the canine trachea. J Appl Physiol 44:137-143

9. Mortola JP, Sant'Ambrogio G 1979 Mechanics of the trachea and behavior of its slowly adapting stretch receptors. J Physiol 286:577-590

10. Kannan MS, Daniel EE 1980 Structural and functional study of control of canine tracheal smooth muscle. Am J Physiol 238:C27-33

11. Northway WA, Rosan RC, Porter DY 1967 Pulmonary disease following respiratory therapy of hyaline membrane disease: bronchopulmonary dysplasia. N Engl J Med 276:357-368

12. Olsen CR, Stevens AE, Mcllroy MB 1967 Rigidity of trachea and bronchi during muscular constriction. J Appl Physiol 23:27-34

13. Hanks BSR, Stephens NL 1981 Mechanics and energetics of lengthening of active airway smooth muscle. Am J Physiol 241:C42-46

14. Jones JG, Fraser RB, Nadel JA 1975 Effect of changing airway mechanics on maximum expiratory flow. J Appl Physiol 38:10-12

15. Olsen CR, Stevens AE, Pride NB, Staub NC 1967 Structural basis for decreased compressibility of constricted trachae and bronchi. J Appl Physiol 23:35-39

16. Knudson RJ, Knudson DE 1975 Effect of muscle constriction on flow-limiting collapse of isolated canine trachea. J Appl Physiol 38:125-131 